Escarbajal de Haro, Andrés; Martínez de Miguel López, Silvia Margarita; Salmerón Aroca, Juan Antonio (2015). La percepción de la calidad de vida en las mujeres mayores y su envejecimiento activo a través de actividades socioeducativas en los centros sociales. Revista de Investigación Educativa, 33(2), 471-488.

DOI: http://dx.doi.org/10.6018/rie.33.2.213211

\title{
La percepción de la calidad de vida en las mujeres mayores y su envejecimiento activo a través de actividades socioeducativas en los centros sociales
}

\author{
Perception of the quality of life in older \\ women and their active ageing through socio educational \\ activities in the social centers
}

\author{
Andrés Escarbajal de Haro, Silvia Margarita Martínez de Miguel López \\ y Juan Antonio Salmerón Aroca \\ Universidad de Murcia
}

\begin{abstract}
Resumen
En el presente artículo se da cuenta de una investigación sobre la percepción de la calidad de vida en las mujeres mayores usuarias de los centros sociales, y la importancia de participar en actividades socioculturales para un envejecimiento activo, así como sus opiniones acerca del modelo educativo más adecuado a sus intereses y motivaciones vitales. Se ha llevado a cabo en centros sociales de los municipios que conforman el valle de Ricote de la Región de Murcia. A todas las participantes se les administró la escala de valoración psicoafectiva Yesavage (GDS) y el cuestionario sobre estereotipos negativos hacia la vejez CENVE. Una vez obtenidos los resultados, fueron analizados en varios grupos de discusión. Se seleccionaron inicialmente 77 participantes; no obstante hubo "muerte experimental" de 13 usuarias, por lo que la muestra definitiva se conformó con 64 usuarias. El análisis estadístico se realizó con el paquete estadístico SPSS v. 15.0. Respecto a la percepción que tienen de sí mismas expresan un grado de satisfacción elevado con la manera en que transcurren sus vidas, aunque existen diferencias interindividuales. En lo que concierne a la vivencia de su participación en centros sociales, se puede señalar que las actividades socioeducativas desarrolladas hacen
\end{abstract}


que el asociacionismo se vaya asentando entre las mujeres mayores. También presentan una actitud abierta hacia nuevos aprendizajes, mostrando preferencia por las actividades de grupo.

Palabras clave: mujeres mayores, calidad de vida, asociacionismo, actividades socioeducativas.

\section{Abstract}

This article presents a study on the perception of quality of life among female users of social institutions, and the relevance for taking part in sociocultural activities for an active ageing, as well as womens' opinions about the most suitable educational model according to their interests and motivations. The study was conducted in social institutions located in the valley of Ricote in the Region of Murcia (Spain). Participants completed the GDS scale (psychoaffective rating scale) and CENVE (questionnaire about negative stereotypes towards aging). Results were analyzed in several discussion groups. Seventy seven women were initially selected, yet only 64 made up the final sample. Statistics analyses were conducted with SPSS v.15.0. In terms of participants' experience in social institutions, results show that social and educational activities are well established among women. Moreover, they have an open attitude toward learning and prefer group activities.

Keywords: mature women, quality of life, associations, social and educational activities.

\section{Introducción}

La mayor longevidad de la población está considerada como una de las características definitorias del presente siglo XXI (Pérez Díaz, 2010). En el contexto europeo, además, subyace un concepto de envejecimiento activo que encierra la idea de que envejecer supone, más que la aceptación de las disfunciones y alteraciones asociadas al avance de la edad, la optimización de las potencialidades y posibilidades de crecimiento y desarrollo personal. Igualmente, se debe destacar que en la población de personas mayores se está dando un creciente desequilibrio de género a medida que aumenta la edad, con lo que se está produciendo una feminización del envejecimiento (IMSERSO, 2011). En España las mujeres mayores (casi el 10\% de la población) presentan mayor esperanza de vida que sus generaciones precedentes y diferencialmente respecto a los hombres: 85.4 años de vida por término medio para mujeres y 79.5 para hombres (INE, 2014). Sin embargo, los estudios de género en mayores son relativamente recientes en nuestro país, pues hasta finales de los 80 del pasado siglo existía escasa bibliografía al respecto (Alcain, Rubio y Sevillano, 2003); de este modo, "la invisibilidad de la mujer, es la nota dominante en la bibliografía sobre envejecimiento, y gerontología, así como la escasez de estudios e investigaciones referentes al tema" (Serdio, 2006, p. 14).

En cuanto a la relación que existe entre la percepción personal del envejecimiento y la longevidad, hay evidencias acerca de que las percepciones, actitudes y pensamientos positivos en los mayores aumentan sus años de vida. Varios estudios (Barefoot, Maynard, Beckham, Brummet, Hooker \& Siegler, 1998; Hamarat, Thompson, Steele, Matheny \& Simmons, 2002; Levy, 2002; Moor, Zimprich, Schmitt, \& Kliegel, 2006) demuestran la existencia de correlación entre ambas variables, así como la posibilidad de modificarlas con una adecuada intervención socioeducativa. 
Por esa razón, el trabajo de investigación que a continuación se expone se ha centrado en las vivencias de envejecer, expresadas en forma de sentimientos y valoraciones generales ante la vida, de percepciones personales del proceso evolutivo y de aspectos vitales que pueden acontecer en la vejez y en su repercusión para la educación, ya que tener en cuenta las creencias, expectativas y atribuciones es básico para los planteamientos educativos con las personas mayores (Vega, Buz y Bueno, 2002).

En la investigación se ha constatado lo que otros autores estudiaron hace años: la importancia de afrontar la etapa de la vejez a través de la participación ciudadana en centros sociales donde se pueden desarrollar actividades socioeducativas (Barnés, 2005; Puigvert, 2005). En esos centros se ponen en juego procesos de dinamización e implicación en proyectos de diversa índole, pero también se percibe, en general, que en el caso de las mujeres mayores asociadas todavía hay un porcentaje muy significativo que no se atreven a alzar la voz para opinar o explicar su postura, que se instalan fácilmente en las inercias, rutinas y estereotipos de las prácticas sociales tradicionales.

Por los motivos anteriores, el estudio que se presenta aborda un análisis descriptivo y exploratorio de cómo perciben las mujeres mayores su propia situación. Se ha desarrollado revisando la bibliografía más destacada existente al respecto y en base a una investigación empírica, complementada con el desarrollo de grupos de discusión, en mujeres mayores usuarias de centros sociales de los municipios que conforman el valle de Ricote de la Región de Murcia. El motivo de circunscribirlo a ese entorno geográfico obedece a que registra la mayor tasa de longevidad de la Región de Murcia.

\section{Marco teórico}

El envejecimiento como tal no será bien entendido hasta que dejen de prevalecer las connotaciones negativas asentadas en esquemas biologicistas del siglo pasado no superados todavía, identificando erróneamente el aumento de la edad con procesos de deterioro, síntomas de decadencia, desaceleración, pérdida de vigor, etc. Tampoco, como señala Pérez Díaz (2006), han ayudado, en absoluto, las previsiones catastrofistas acerca del aumento demográfico de mayores, donde se vaticinaba un futuro problemático causado por el crecimiento de la franja de edad de personas entre los 65 y 85 años, o como consecuencia de ello. Se habla concretamente de factores socioeconómicos como el gasto en pensiones y el aumento de la relación de dependencia. Sin embargo, no es infrecuente que se obvien o se trivialicen los roles tan importantes que están llevando a cabo los mayores en nuestra sociedad, además del soporte económico que suponen para las familias en momentos de crisis económica

Por consiguiente, el proceso de envejecimiento no ha de ser observado como un problema, sino como un desafío para todos, tanto para la sociedad como para el individuo que envejece (Abellán y Esparza, 2009; Meléndez, Navarro, Oliver, y Tomás, 2009). Y en ese desafío, algunas voces destacadas en el terreno de la educación de mayores (Montero, García y Bedmar, 2011) han señalado la necesidad e importancia 
de enfoques socioeducativos en los centros de mayores; de hecho, es también una de las demandas de las mujeres mayores del estudio, como se verá en los resultados y conclusiones.

En otro orden de cosas, Levy y Myers (2003), tras realizar varios estudios experimentales y longitudinales en personas mayores, concluyeron que los estereotipos negativos sobre la vejez influyen en su memoria, causan estrés y peores estrategias para combatirlos, e incluso, predicen menor longevidad. También demostraron que aquellos mayores que tenían una opinión positiva sobre su propio envejecimiento gozaron (en un seguimiento de 18 años) de una salud funcional mejor que los que tenían una opinión más negativa. En este sentido, y en relación a las mujeres mayores en España, en general, una gran parte de ellas percibe su situación de envejecimiento de forma positiva, según se desprende de un estudio del IMSERSO (2011); la mitad se siente muy o bastante satisfecha con su situación actual, un $90 \%$ se encuentra satisfecha con sus relaciones familiares, y un $78.8 \%$ con sus relaciones de amistad; y se sienten seguras y confiadas con la gente que les rodea. Al respecto, es interesante la aportación de Moor et al. (2006), cuando afirman que es posible realizar modificaciones en las actitudes negativas del envejecimiento; los resultados de su investigación indican que las actitudes negativas sobre el propio envejecimiento se pueden modificar con intervenciones socioeducativas.

Otra investigación, de Urquijo, Monchietti y Krzemien (2008), concluyó que aquellas mujeres mayores que utilizaban estrategias de afrontamiento activas, cognitivas y conductuales mejoran su calidad de vida y exploran alternativas de acción para superar los obstáculos que se les presenta en la vida; en cambio, aquellas que poseen estilos de personalidad caracterizados por preservación, acomodación y protección, suelen interesarse en atender las necesidades de los demás y se muestran prudentes, reservadas y sin intención de intervenir activamente en el curso de los hechos.

En cuanto a la calidad de vida, existe consenso al aceptar que ese concepto presenta un carácter multidimensional. Si en la década de los sesenta del siglo XX se editaban artículos sobre satisfacción con la vida, años después aparece el tema de envejecimiento con éxito; en los setenta surge el concepto de calidad de vida; y más tarde, a partir de los ochenta, el de bienestar subjetivo se convierte en dominante (Fernández Ballesteros, 2009). Actualmente, es de interés el concepto de envejecimiento activo, que es generalmente aceptado como un proceso de adaptación a través del cual se logra un óptimo desarrollo físico, psicológico y social(Rodríguez, 2013).

De ese modo, 2012 fue declarado como «Año europeo del envejecimiento activo y de la solidaridad intergeneracional» con el objetivo de promover la creación en Europa de un envejecimiento saludable en el marco de una sociedad para todas las edades. Además, por iniciativa del gobierno de España,se publicó el Libro blanco del envejecimiento activo (2011) para orientar las políticas dirigidas a mejorar la calidad de vida de las personas mayores. En él se recogen los determinantes que favorecen el envejecimiento activo: economía, salud, actividad física, educación a lo largo de la vida, participación, etc. La acepción activo se refiere a la participación continua en las cuestiones sociales, económicas, culturales..., y no solamente a la capacidad de estar físicamente activo. Ello requiere el ejercicio del control vital, que puede ser considerado como "el producto del proceso de adaptación que ocurre a lo largo de 
la vida, a través del cual se logra un óptimo desarrollo físico (incluyendo la salud), psicológico (óptimo funcionamiento cognitivo y autorregulación emocional) y social del individuo" (Fernández Ballesteros, 2009, p. 182).

No obstante, para conseguir ese propósito es ineludible tener una visión positiva del envejecimiento (Lehr, 2009); y, para tenerla, la educación y la participación social son elementos esenciales (Villaplana, 2010). De tal manera, que ese argumento se reveló primordial para el desarrollo de esta investigación; perspectiva que entronca directamente con la posibilidad de "cambio" en cualquier momento de la vida, donde las personas mayores son consideradas con capacidades y recursos, "sujetos del futuro, protagonistas activos, dispuestos a redefinirse a sí mismos mediante su capacidad de ser mentalmente abiertos a nuevas experiencias, a los cambios y a las oportunidades que puedan desarrollar ellos mismos" (Escotorin y Roche, 2011, p. 18).

En todo caso, no se trata de "prescribir" ni de "imponer" un patrón único de envejecimiento activo, dada la heterogeneidad que supone el envejecimiento y la variabilidad que existe en la manera de envejecer de una persona a otra; de tal forma que se debe destacar que la acción educativa se tiene que desarrollar de modo consensuado, reflexivo, dialógico, partiendo de las necesidades de los mayores y respetando su autonomía.

\section{Objetivos}

Los objetivos propuestos en la investigación fueron:

a) Describir a las mujeres mayores que están participando en centros sociales del valle de Ricote de la Región de Murcia.

b) Caracterizar la percepción que tiene ese colectivo sobre vejez y calidad de vida.

c) Analizar sus vivencias en procesos de participación socioeducativa.

d) Recabar sus opiniones sobre las actividades socioeducativas en las que participan para valorar el modelo educativo más adecuado para ellas.

\section{Metodología}

La población de estudio se enmarca en la denominada Comarca del valle de Ricote de la Región de Murcia. En esa zona hay censadas 12.292 personas mayores de 65 años, lo que supone un $15.7 \%$ de su población total (de los cuales el $56.9 \%$ corresponde a mujeres y el $43.1 \%$ corresponde a hombres). Estos datos no son diferentes en cuanto a la feminización del envejecimiento tanto a nivel nacional como regional; ahora bien, en cuanto al índice de vejez, que es el indicador que mide la proporción de mayores de 65 años respecto a los menores de 20 años, esta zona presenta una ratio muy elevada. Esto hace que se convierta en la zona más envejecida de la Región de Murcia. Los datos proporcionados tanto por la Comunidad Autónoma de la Región de Murcia (CARM, 2012) como los aparecidos en el Boletín Epidemiológico de la Región de Murcia (2010), y que pueden observarse en la tabla 1, ilustran que esta zona geográfica presenta los registros más elevados de esperanza de vida femenina de toda la Región de Murcia. 
Tabla 1

Población de mayores de 65 años del valle de Ricote

\begin{tabular}{ccccc}
\hline Municipio & $\begin{array}{c}\text { No mayores }>65 \\
\text { años (ambos } \\
\text { sexos) }\end{array}$ & $\begin{array}{c}\text { No mujeres } \\
\text { mayores }>65 \\
\text { años }\end{array}$ & $\begin{array}{c}\text { \% de mujeres } \\
\text { mayores }>65 \\
\text { años }\end{array}$ & $\begin{array}{c}\text { Esperanza de } \\
\text { vida media } \\
\text { (años) }\end{array}$ \\
\hline Abarán & 2.194 & 1.240 & $56.52 \%$ & 81.1 \\
Archena & 2.641 & 1.4 .60 & $55.28 \%$ & 80.5 \\
Blanca & 1.180 & 685 & $58.05 \%$ & 82.8 \\
Cieza & 5.401 & 3.140 & $58.14 \%$ & 80.6 \\
Ojós & 117 & 71 & $60.68 \%$ & 80.0 \\
Ricote & 364 & 209 & $57.42 \%$ & 82.3 \\
Ulea & 224 & 122 & $54.46 \%$ & 80.8 \\
Villanueva & 415 & 236 & $60.51 \%$ & 78.8 \\
\hline
\end{tabular}

Fuente: Elaboración propia a partir de las fuentes citadas.

Teniendo en cuenta ese contexto, la investigación que se presenta, de carácter exploratorio-descriptivo, se orientaba al estudio del proceso de envejecimiento, buscando las claves diferenciadoras del envejecimiento femenino y su relación con el proceso de participación socioeducativa. Se llevó a cabo la administración de escalas y tests con fines descriptivos. A todas las participantes se les administró una escala de valoración psicoafectiva, así como el cuestionario sobre estereotipos negativos hacia la vejez. Una vez obtenidos los resultados, fueron analizados en varios grupos de discusión para intentar clarificarlos, matizarlos y completarlos.

La escala de valoración del estado psicoafectivo en mayores, conocida con GDS o Escala de Depresión Geriátrica de Yesavage, consta de quince ítems, con respuesta sí/ no y validada en población española para el cribado de la depresión en mayores de 65 años. El punto de corte para el diagnóstico de posible depresión se sitúa en una puntuación de 5. Presenta una sensibilidad del $81.1 \%$ y una especificidad del $76.7 \%$ (Martínez de la Iglesia et al, 2002). No obstante, diversos autores recomiendan utilizar un punto de corte superior a 10 (sensibilidad del 80\% y una especificidad del 100\%) (Agüera, Cervilla y Martín, 2006).

El cuestionario de estereotipos negativos hacia la vejez (CENVE), diseñado por Sánchez Palacios (2004), está compuesto por 15 ítems que saturan en tres factores: salud, motivacional-social y carácter-personalidad, cuyo formato de respuesta sigue un modelo tipo Likert de cuatro escalones. Según los datos proporcionados por la propia autora, la varianza explicada para estos tres factores es del 56.96\%. Los resultados de la prueba KMO fueron de .88 y la prueba de esfericidad de Barlett obtuvo 
una significación de $\mathrm{p}<0.001$. Respecto al análisis de la fiabilidad de cada uno de los factores obtuvo valores entre .64 y .67 al aplicar el alfa de Cronbach.

Los criterios de selección para el estudio fueron los siguientes: mujeres de 65 años o más, residentes en los municipios de la comarca del valle de Ricote, no vivir en residencias o centros institucionales, pertenecer a alguna de las asociaciones o centros sociales de la zona y encontrarse realizando actividades socioeducativas en el momento de la investigación. Fueron seleccionadas inicialmente 77 participantes que cumplían con esos criterios, aunque hubo "muerte experimental" de 13 usuarias, por lo que la muestra definitiva se conformó con 64 usuarias de 8 centros sociales.

La escala y el cuestionario utilizados fueron corregidos y puntuados siguiendo las normas propuestas por los autores de los mismos. Estas puntuaciones fueron organizadas y volcadas en una base de datos diseñada específicamente para tal fin a través del programa de Microsoft, Excell 2007 previo al análisis estadístico. El análisis estadístico se realizó con el paquete estadístico SPSS v. 15.0 para Windows. Para el procesamiento estadístico se analizaron los porcentajes y frecuencias de respuesta de los ítems de las variables categóricas establecidas. Además se analizaron las relaciones entre los ítems del CENVE con las categorías comunes de los datos sociodemográficos, empleándose pruebas de chi-cuadrado.

Para complementar la consecución de los objetivos propuestos en este trabajo, también se procedió al desarrollo de 8 grupos de discusión en los 8 centros sociales finalmente seleccionados, tal y como se indicaba en párrafos anteriores. Básicamente el análisis de contenido se realizó mediante procesos de teorización (exploración, descripción e interpretación de los datos), procedimientos analíticos generales (mediante reducción y exposición de los datos mediante categorías descriptivas) y estrategias de selección secuencial para generar constructos (Lecompte y Goetz, 1982).

\section{Resultados}

Las edades de las integrantes de la muestra oscilan entre 65 y 84 años, siendo la media de edad de 70.5 años. Por franjas etarias la muestra se estructuraba en: 31 mujeres sexagenarias, 30 septuagenarias y 3 octogenarias. Respecto al estado civil de la muestra queda recogido en el cuadro siguiente:

Tabla 2

Distribución del estado civil

\begin{tabular}{cccc}
\hline Soltera & Casada & Separada & Viuda \\
\hline$(6)$ & $(39)$ & $(1)$ & $(18)$ \\
\hline
\end{tabular}

Mayoritariamente, las participantes de la muestra están casadas (60.9\%); aproximadamente $1 / 3$ de la muestra son viudas; siendo las situaciones de soltería $(9.3 \%)$ y separación (1.5\%). En cuanto a las profesiones desarrolladas en su vida: 
Tabla 3

Diferenciación laboral de las participantes

\begin{tabular}{cccc}
\hline Ama de casa & $\begin{array}{c}\text { Ama de casa } \\
+\end{array}$ & $\begin{array}{c}\text { Ama de casa } \\
+ \\
\text { Hortofrutícola }\end{array}$ & $\begin{array}{c}\text { Ama de casa } \\
+ \\
\text { Servicios }\end{array}$ \\
\hline$(20)$ & $(28)$ & $(10)$ & $(6)$ \\
\hline
\end{tabular}

Domina el sector hortofrutícola (43.9\%), seguido del sector servicios (15.9\%); y, en menor porcentaje,había participantes que fueron empresarias autónomas $(9.3 \%)$ con pequeños comercios o en el sector hostelero.

En cuanto al nivel de formación adquirido, la mayor parte realizaron estudios primarios, y es escaso el porcentaje de las que cursaron estudios medios o superiores (4\%). Un $10 \%$ de la muestra no tuvieron la suficiente continuidad en la formación, lo que las hace reconocerse con estudios inferiores a los primarios.

Tabla 4

Nivel de instrucción alcanzado

\begin{tabular}{ccc}
\hline$<$ Primarios & Estudios primarios & Estudios Medios o Sup. \\
\hline$(7)$ & $(54)$ & $(3)$ \\
\hline
\end{tabular}

En referencia al modo de convivencia, se obtuvieron los siguientes datos:

Tabla 5

Forma de convivencia de las participantes

\begin{tabular}{ccc}
\hline En casa - sola & En casa - acompañada & En casa de un familiar \\
\hline$(23)$ & $(41)$ & $(0)$ \\
\hline
\end{tabular}

Se puede observar cómo mayoritariamente viven acompañadas en su casa (64\%), y en menor medida solas.

En cuanto al rol de cuidadora que desempeñan, el siguiente cuadro refleja los datos recogidos:

Tabla 6

Personas a su cargo

\begin{tabular}{cccc}
\hline Enfermo o discapacitado & Hijo & Nieto & Nadie \\
\hline$(6)$ & $(12)$ & $(10)$ & $(36)$ \\
\hline
\end{tabular}


La principal dedicación en su rol de cuidadoras se dirige a los hijos (19\%), seguida de los nietos (16\%) y de personas que presentan alguna enfermedad o discapacidad (9\%). Más de la mitad de la muestra (56\%) no eran cuidadoras principales en ese momento.

Por último, referente a los años de participación en los centros sociales, se obtuvieron los siguientes datos:

Tabla 7

Años de participación socio-comunitaria

\begin{tabular}{cccccccc}
\hline $0-5$ & $6-10$ & $11-15$ & $16-20$ & $21-25$ & $26-30$ & $31-35$ & $>36$ \\
\hline$(20)$ & $(32)$ & $(8)$ & $(2)$ & $(0)$ & $(0)$ & $(2)$ & $(0)$ \\
\hline
\end{tabular}

De esos datos se desprende que el 50\% llevaba al menos 6 años en los centros, el $29 \%$ cerca de 11 años, y el 31\% de participantes en el estudio llevaba hasta cinco años participando.

Se presentan seguidamente los resultados obtenidos para la valoración del estado anímico y afectivo tras la administración de la escala Yesavage-15 (tabla 8) con el formato de frecuencias y porcentajes, a través de tabla y figura.

En los ítems de la escala relacionados con la satisfacción en el transcurso de sus vidas (ítems 1, 3, 7, 11) cabe decir que los datos obtenidos muestran unos valores elevados, con una media de $86.35 \%$; respecto a los datos que se pueden relacionar con su estado de salud (ítems 10 y 15), el 85.9\% de la muestra refiere no tener más problemas de memoria que el resto y sentirse mejor que otras personas (84.4\%). También los ítems 5, 12 y 14, referidos a estado de ánimo, utilidad y esperanza respectivamente, muestran buenos índices entre las mujeres mayores, con una media de aceptación de $82.3 \%$ para los tres items.

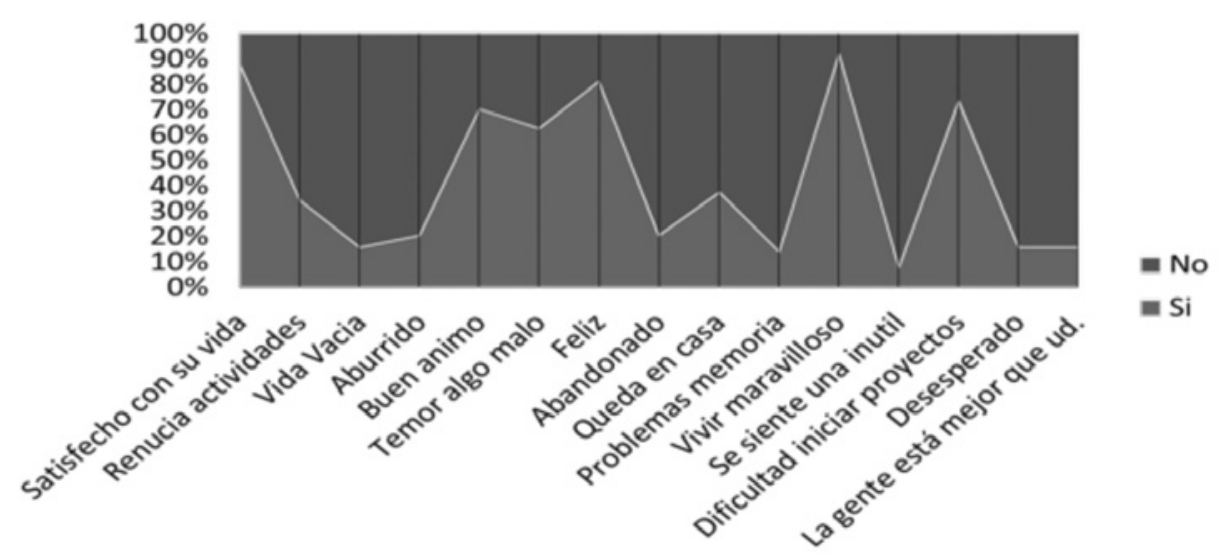

Figura 1. Respuestas Yesavage-15. 
Tabla 8

Frecuencia y porcentaje de respuesta de la escala (YESAVAGE-15)

\begin{tabular}{|c|c|c|c|c|c|}
\hline & \multirow[b]{2}{*}{ Elementos } & \multicolumn{2}{|c|}{ Frecuencia } & \multicolumn{2}{|c|}{ Porcentaje } \\
\hline & & Sí & No & Sí & No \\
\hline 1. & ¿Está satisfecho con su vida? & 56 & 8 & $87.5 \%$ & $12.5 \%$ \\
\hline 2. & ¿Ha renunciado a muchas actividades? & 22 & 42 & $34.4 \%$ & $65.6 \%$ \\
\hline 3. & ¿Siente que su vida está vacía? & 10 & 54 & $15.6 \%$ & $84.4 \%$ \\
\hline 4. & ¿Se encuentra a menudo aburrido/a? & 13 & 51 & $20.3 \%$ & $79.7 \%$ \\
\hline 5. & ¿Tiene a menudo buen ánimo? & 45 & 19 & $70.3 \%$ & $29.7 \%$ \\
\hline 6. & ¿Teme que le pase algo malo? & 40 & 24 & $62.5 \%$ & $37.5 \%$ \\
\hline 7. & ¿Se siente feliz muchas veces? & 52 & 12 & $81.3 \%$ & $18.8 \%$ \\
\hline 8. & ¿Se siente a menudo abandonado/a? & 13 & 51 & $20.3 \%$ & $79.7 \%$ \\
\hline 9. & ¿Prefiere quedarse en casa a salir? & 24 & 40 & $37.5 \%$ & $62.5 \%$ \\
\hline 10 & ¿Cree tener más problemas de memoria que el resto de la gente? & 9 & 55 & $14.1 \%$ & $85.9 \%$ \\
\hline 11. & ¿Piensa que es maravilloso vivir? & 59 & 5 & $92.2 \%$ & $7.8 \%$ \\
\hline 12. & ¿Actualmente se siente una inútil? & 5 & 59 & $7.8 \%$ & $92.2 \%$ \\
\hline 13. & ¿Le cuesta iniciar nuevos proyectos? & 47 & 17 & $73.4 \%$ & $26.6 \%$ \\
\hline 14. & ¿Siente que su situación es desesperada? & 10 & 54 & $15.6 \%$ & $84.4 \%$ \\
\hline 15. & ¿Cree que mucha gente está mejor que usted? & 10 & 54 & $15.6 \%$ & $84.4 \%$ \\
\hline
\end{tabular}

Frente a los datos optimistas, también hubo algunas puntuaciones en sentido contrario: hay un porcentaje considerable de mujeres mayores $(20.3 \%)$ que se sienten aburridas y abandonadas (ítems 4 y 8). El ítem que con mayor frecuencia fue respondido en sentido negativo fue el que hace referencia a la dificultad para iniciar nuevos proyectos (ítem 13), con el $73.4 \%$ de la muestra. También llama la atención el alto porcentaje obtenido respecto a la creencia generalizada de que algo malo pueda suceder en sus vidas (ítem 6), donde más de la mitad responde afirmativamente (62.5\%). De la misma manera, el $34,4 \%$ opina que habían renunciado a muchos de sus intereses y actividades anteriores (ítem 2), así como que preferían quedarse en casa a salir a la calle (37.5\%) (ítem 9).

Para la medición de los estereotipos negativos se utilizó el cuestionario, anteriormente comentado, de Sánchez Palacios (2004). El 98.4\% de la muestra presentó algún estereotipo negativo en sus respuestas. Entre los estereotipos del cuestionario, aquellos que se presentaron con más frecuencia en la muestra fueron los que hacen referencia al estado físico y la salud (57.7\%), seguidos de los que hacen referencia al carácter y la personalidad (50.3\%) y de los referidos a las motivaciones y vida social $(48.78 \%)$. Hay también hay una serie de ítems del cuestionario $(4,5,6,8,10,14,15)$ que, si bien estuvieron presentes en las respuestas de algunos informantes, no fueron señalados por la mayoría de la muestra. La tabla 9 refleja el análisis de los resultados diferenciados para cada ítem del cuestionario. 
Tabla 9

Frecuencias y porcentajes de respuesta en los items del CENVE $(N=64)$

\begin{tabular}{|c|c|c|c|c|c|c|c|c|c|}
\hline & \multicolumn{9}{|c|}{ Puntuación } \\
\hline & Elementos & 1 & 2 & 3 & 4 & Mediana & Moda & Media & DT \\
\hline 1. & $\begin{array}{l}\text { La mayor parte de las personas, cuando } \\
\text { llegan a los } 65 \text { años de edad, aproximada- } \\
\text { mente, comienzan a tener un considerable } \\
\text { deterioro de la memoria }\end{array}$ & $\begin{array}{c}(10) \\
15.6 \%\end{array}$ & $\begin{array}{c}(5) \\
7.8 \%\end{array}$ & $\begin{array}{c}(22) \\
34.4 \%\end{array}$ & $\begin{array}{c}(27) \\
42.4 \%\end{array}$ & 3 & 4 & 3.03 & 1.06 \\
\hline 2. & $\begin{array}{l}\text { Las personas mayores tienen menos interés } \\
\text { por el sexo }\end{array}$ & $\begin{array}{c}(11) \\
17.2 \%\end{array}$ & $\begin{array}{c}(10) \\
15.6 \%\end{array}$ & $\begin{array}{c}(26) \\
40.6 \%\end{array}$ & $\begin{array}{c}(17) \\
26.6 \%\end{array}$ & 3 & 3 & 2.76 & 1.03 \\
\hline 3. & $\begin{array}{l}\text { Las personas mayores se irritan con facili- } \\
\text { dad y son "cascarrabias" }\end{array}$ & $\begin{array}{l}(16) \\
25 \%\end{array}$ & $\begin{array}{c}(15) \\
23.4 \%\end{array}$ & $\begin{array}{l}(16) \\
25 \%\end{array}$ & $\begin{array}{c}(17) \\
26.6 \%\end{array}$ & 3 & 4 & 2.53 & 1.14 \\
\hline 4. & $\begin{array}{l}\text { La mayoría de las personas mayores de } \\
70 \text { años tienen alguna enfermedad mental } \\
\text { lo bastante seria como para deteriorar sus } \\
\text { capacidades normales }\end{array}$ & $\begin{array}{c}(20) \\
31.3 \%\end{array}$ & $\begin{array}{c}(19) \\
29.7 \%\end{array}$ & $\begin{array}{c}(11) \\
17.2 \%\end{array}$ & $\begin{array}{c}(14) \\
21.9 \%\end{array}$ & 2 & 1 & 2.29 & 1.13 \\
\hline 5. & $\begin{array}{l}\text { Las personas mayores tienen menos amigos } \\
\text { que las más jóvenes }\end{array}$ & $\begin{array}{c}(19) \\
29.7 \%\end{array}$ & $\begin{array}{c}(15) \\
23.4 \%\end{array}$ & $\begin{array}{l}(16) \\
25 \%\end{array}$ & $\begin{array}{c}(14) \\
21.9 \%\end{array}$ & 2 & 1 & 2.39 & 1.13 \\
\hline 6. & $\begin{array}{l}\text { A medida que las personas mayores se } \\
\text { hacen mayores, se vuelven más rígidas }\end{array}$ & $\begin{array}{c}(17) \\
26.6 \%\end{array}$ & $\begin{array}{c}(18) \\
28.1 \%\end{array}$ & $\begin{array}{c}(18) \\
28.1 \%\end{array}$ & $\begin{array}{c}(11) \\
17.2 \%\end{array}$ & 2 & 2 & 2.35 & 1.05 \\
\hline 7. & $\begin{array}{l}\text { La mayor parte de los adultos mantienen un } \\
\text { nivel de salud aceptable hasta los } 65 \text { años } \\
\text { aproximadamente, en donde se produce un } \\
\text { fuerte deterioro de la salud }\end{array}$ & $\begin{array}{c}(10) \\
15.6 \%\end{array}$ & $\begin{array}{c}(13) \\
20.3 \%\end{array}$ & $\begin{array}{c}(22) \\
34.4 \%\end{array}$ & $\begin{array}{c}(19) \\
29.7 \%\end{array}$ & 3 & 3 & 2.78 & 1.04 \\
\hline 8. & $\begin{array}{l}\text { A medida que nos hacemos mayores perde- } \\
\text { mos el interés por las cosas }\end{array}$ & $\begin{array}{c}(23) \\
35.9 \%\end{array}$ & $\begin{array}{c}(13) \\
20.3 \%\end{array}$ & $\begin{array}{c}(19) \\
29.7 \%\end{array}$ & $\begin{array}{c}(9) \\
14.1 \%\end{array}$ & 2 & 1 & 2.21 & 1.09 \\
\hline 9. & $\begin{array}{l}\text { Las personas mayores son, en muchas } \\
\text { ocasiones, como niños }\end{array}$ & $\begin{array}{c}(14) \\
21.9 \%\end{array}$ & $\begin{array}{c}(7) \\
10.9 \%\end{array}$ & $\begin{array}{c}(23) \\
35.9 \%\end{array}$ & $\begin{array}{c}(20) \\
31.3 \%\end{array}$ & 3 & 4 & 2.76 & 1.12 \\
\hline 10 & $\begin{array}{l}\text { La mayor parte de las personas mayores de } \\
65 \text { años tienen una serie de incapacidades } \\
\text { que les hacen depender de los demás }\end{array}$ & $\begin{array}{c}(21) \\
32.8 \%\end{array}$ & $\begin{array}{c}(19) \\
29.7 \%\end{array}$ & $\begin{array}{c}(17) \\
26.6 \%\end{array}$ & $\begin{array}{c}(7) \\
10.9 \%\end{array}$ & 2 & 1 & 2.15 & 1.01 \\
\hline 11. & $\begin{array}{l}\text { A medida que nos hacemos mayores perde- } \\
\text { mos la capacidad de resolver los problemas } \\
\text { a los que nos enfrentamos }\end{array}$ & $\begin{array}{c}(12) \\
18.8 \%\end{array}$ & $\begin{array}{l}(18) \\
8.1 \%\end{array}$ & $\begin{array}{c}(20) \\
31.3 \%\end{array}$ & $\begin{array}{c}(14) \\
21.9 \%\end{array}$ & 3 & 3 & 2.56 & 1.03 \\
\hline 12. & $\begin{array}{l}\text { Los defectos de la gente se agudizan con } \\
\text { la edad }\end{array}$ & $\begin{array}{c}(14) \\
21.9 \%\end{array}$ & $\begin{array}{c}(11) \\
17.2 \%\end{array}$ & $\begin{array}{c}(18) \\
28.1 \%\end{array}$ & $\begin{array}{c}(21) \\
32.8 \%\end{array}$ & 3 & 4 & 2.71 & 1.14 \\
\hline 13. & $\begin{array}{l}\text { El deterioro cognitivo (pérdida de memoria, } \\
\text { desorientación, confusión) es una parte } \\
\text { inevitable de la vejez }\end{array}$ & $\begin{array}{c}(12) \\
18.8 \%\end{array}$ & $\begin{array}{c}(13) \\
20.3 \%\end{array}$ & $\begin{array}{c}(19) \\
29.7 \%\end{array}$ & $\begin{array}{c}(20) \\
31.3 \%\end{array}$ & 3 & 4 & 2.73 & 1.10 \\
\hline 14. & $\begin{array}{l}\text { Casi ninguna persona mayor de } 65 \text { años } \\
\text { realiza un trabajo tan bien como lo haría otra } \\
\text { más joven }\end{array}$ & $\begin{array}{c}(24) \\
37.5 \%\end{array}$ & $\begin{array}{c}(19) \\
29.7 \%\end{array}$ & $\begin{array}{c}(13) \\
20.3 \%\end{array}$ & $\begin{array}{c}(8) \\
12.5 \%\end{array}$ & 2 & 1 & 2.07 & 1.04 \\
\hline 15. & $\begin{array}{l}\text { Una gran parte de las personas mayores de } \\
65 \text { años "chochean" }\end{array}$ & $\begin{array}{c}(30) \\
46.9 \%\end{array}$ & $\begin{array}{c}(17) \\
26.6 \%\end{array}$ & $\begin{array}{c}(7) \\
10.9 \%\end{array}$ & $\begin{array}{c}(10) \\
15.6 \%\end{array}$ & 2 & 1 & 1.95 & 1.10 \\
\hline
\end{tabular}

Notas: $1=$ Muy en desacuerdo; 2=Bastante en desacuerdo; 3=Bastante de acuerdo; $4=$ Muy de acuerdo

En los ítems que se relacionan con el factor salud (ítems 1, 4, 7, 10,13) se obtuvieron los siguientes resultados: el $77 \%$ de las mujeres mayores del estudio creen que 
inevitablemente en la vejez hay un empeoramiento de la memoria (ítem 1), el 64\% sostienen que en la vejez se da un deterioro general de la salud (ítems 7), y un 61\% de las participantes afirman que hay un deterioro cognitivo inevitable (ítem 13).

En cuanto a los estereotipos referidos al factor motivacional-social (ítems 2, 5, 8, 11, 14), el $67.2 \%$ responden que a medida que se hacen mayores se pierde la capacidad de resolver problemas (ítem 11). En cambio los resultados fueron más optimistas en el ítem 5 (las personas mayores tienen menos amigos que las más jóvenes), el ítem 8 (a medida que se envejece, se pierde el interés por las cosas) y en el ítem 14 (casi ninguna persona mayor de 65 años realiza un trabajo tan bien como lo haría otra más joven).

Finalmente, en los resultados obtenidos en el factor carácter-personalidad (ítems $3,6,9,12,15)$ destacar que el $51.6 \%$ de la muestra creen que los mayores se irritan con facilidad y son "cascarrabias" (ítem 3), el 67.2\% que se comportan "como niños" (ítem 9), mientras que el $60.9 \%$ afirman que los defectos se agudizan a medida que pasan los años (ítem 12).

En los grupos de discusión, además de analizar los datos expuestos, fueron introducidas cuestiones referidas a la educación (ver ANEXO con la guía de cuestiones). La información complementaria que se extrae de los grupos de discusión realizados se podría sintetizar en los siguientes resultados:

En relación a la percepción que poseen de la vejez, en general, como ciclo vital, existen opiniones diversas, pero es destacable el hecho de que el mayor porcentaje de opiniones ofrece respuestas argumentadas y desarrolladas que hacen referencia a capacidades funcionales (autocuidado, independencia, nivel de actividad etc.) aunque también hacen referencia a percepciones de tipo psicológico (estado de ánimo, ilusión, carácter, personalidad, etc.).

En cuanto a la percepción de su propia vejez, los resultados reflejan que la mayoría de las participantes en los grupos de discusión tienen una autopercepción positiva, con una buena valoración de su proceso de envejecimiento. También existe una argumentación positiva de la percepción personal de la vejez en base a la relación que mantienen con la familia y a la valoración que hacen de la misma,

Con respecto a la experiencia participativa propiamente dicha en las actividades de los centros sociales, en primer lugar, cabe decir que la actitud de las entrevistadas es abierta y colaboradora. Incluso, se encuentran de una manera explícita quejas sobre déficit de actividades y una actitud reivindicativa con demanda de más proyectos en los centros, lo que puede reflejar un cambio en su actitud de participación respecto a las concepciones más clásicas conocidas. No obstante, cabe señalar que, mayoritariamente, con estas actividades consiguen objetivos de estimulación, crecimiento personal, experiencias compartidas, intercambio de saberes entre ellas mismas y entre ellas y los educadores... Indican también la necesidad de las mismas, para paliar el déficit de formación que han sufrido, fruto de su historia personal, así como también conceden a la educación beneficios terapéuticos por lo que supone el ejercicio de sus capacidades cognitivas y socioafectivas. En este sentido, los sentimientos asociados a las narraciones de sus experiencias educativas actuales manifiestan un sentimiento de satisfacción.

Por último referente al planteamiento educativo que más se ajusta a sus expectativas, ha sido común en sus discursos encontrar referencias en las que comparan sus experiencias educativas actuales con las que llevaron a cabo cuando fueron jóvenes. 
No obstante narran cambios respecto al modelo clásico de enseñanza y aprendizaje. Los resultados muestran un reconocimiento de sí mismas en sus compromisos vitales, sus deseos por aprender, una reexperimentación de sus vivencias y, en la mayoría de los casos, una reafirmación en el "ahora", y en el "sí mismas". En este sentido, destacan su preferencia por un formato donde tengan la ocasión de participar desde sus esquemas cognitivos y sus experiencias personales, con predilección por el aprendizaje en grupo, señalando la necesidad de un profesional que participe en sus actividades educativas y formativas.

\section{Discusión}

Los resultados expuestos no distan mucho de los obtenidos en otros estudios similares llevados a cabo en la Región de Murcia (Barnés, 2005; Hernández Pedreño, 2000; Martínez de Miguel, 2003; Miñano y Martínez de Miguel (2011). Sin embargo, existen diferencias con otros estudios realizados en entornos geográficos diferentes, como el estudio de Solé et al. (2005) desarrollado en la zona metropolitana de Barcelona, sobre todo en lo referente al nivel educativo, por encima de la media habitual en estas generaciones, por lo que el itinerario laboral y socioeducativo de la muestra se perfila como uno de los hechos causantes de una situación de desigualdad. En este sentido, los resultados refieren la existencia de dependencia económica respecto al varón, datos que son congruentes con otros estudios (Abellán y Esparza, 2009; Pérez Ortiz, 2004), cuyos resultados confirman que es probable que las mujeres experimenten la pobreza en la ancianidad más que los hombres.

Siendo la salud uno de los principales valores al que aspiran las personas mayores, como refleja el estudio realizado por Lorenzo, Millán-Calenti, Lorenzo-López y Maseda (2013), no siempre las mujeres mayores de la muestra refieren actitudes adecuadas hacia los estilos de vida y comportamientos saludables, lo que a la postre puede traducirse en enfermedades. Esta información es concordante con los datos del informe sobre las mujeres mayores del IMSERSO (2011), que reflejan una mayor concurrencia de patologías crónicas en mujeres mayores que en varones.

Otros resultados destacados tienen que ver con la presencia de estereotipos negativos sobre la vejez, datos que encajan con los aportados por Sánchez Palacios (2004). No obstante los datos no confirman resultados de investigaciones previas en los que la visión de los mayores era esencialmente negativa (Triadó y Villar, 1997), sino que la opinión de la muestra al respecto es matizable en función de diferentes dimensiones. Probablemente, si bien el hecho de participar en los centros sociales no es una condición suficiente para que no aparezcan estos estereotipos negativos, si es cierto que se presentan con un perfil valorativo distinto respecto a la población general, tal y como también recogen los resultados de Sánchez Palacios (2004) respecto a la variable participación social.

En cuanto a las estrategias que ponen en marcha las mujeres mayores frente a situaciones de crisis, los resultados son concordantes con los obtenidos por Urquijo, Monchietti y Krzemien (2008). Las estrategias para afrontar estas situaciones son principalmente cognitivas, tanto activas como pasivas, y centradas tanto en la emoción como en el problema. 
En referencia a los argumentos empleados por las mayores de la muestra para participaren los centros sociales, coinciden con otras investigaciones en la importancia del asociacionismo en las personas mayores, como la realizada por Miñano y Martínez de Miguel (2011), o la de Rodríguez (2013). En las mayores de la muestra las motivaciones son numerosas, y destacan entre ellas la participación en actividades socioeducativas; en este sentido, los resultados son concordantes con los del estudio de Barnés (2005), Krzemien, Urquijo y Monchietti (2004) y Serdio (2004).

En relación a las necesidades educativas expresadas en los grupos de discusión, los resultados son similares, en sus líneas generales, a las conclusiones obtenidas en investigaciones como la de Martínez de Miguel y Escarbajal de Haro (2009), que indican tres grandes grupos de necesidades: informativas, formativas/educativas y de ocio y tiempo libre creativo en las mujeres mayores. Los resultados entroncan en la línea de la progresiva demanda de realización de cursos de aprendizaje que se recoge en el estudio de Abellán y Esparza (2009) o los trabajos realizados por Montero, García Mínguez y Bedmar (2011), y que evidencian la elevada motivación que existe en esta área por parte de las participantes.

\section{Conclusiones}

Se puede afirmar que los resultados y discusión expuestos responden a los objetivos específicos propuestos al inicio de la investigación.

Los procesos de socialización que han vivido las mujeres mayores, fruto de un marco histórico, generacional y sociocultural de desigualdad, marcaron de una manera muy clara tanto los aspectos formales de su formación (muy insuficiente) como su dedicación a las actividades laborales (trabajos en el sector primario para ayudar a la economía doméstica). No obstante, las mujeres mayores de la muestra se definen como más activas, participativas y abiertas al aprendizaje que los hombres. Manifiestan bienestar participando en actividades formativas para su desarrollo personal intelectual, social y familiar. Suelen ser solidarias y con un comportamiento prosocial elevado.

Respecto a la imagen, la percepción que tienen de sí mismas expresa un grado de satisfacción elevado con la manera en que transcurren sus vidas, aunque existen diferencias interindividuales respecto a la apreciación cognitiva sobre este período de la vida.

Las mujeres estudiadas muestran una gran preocupación por las enfermedades, porno mantener un estado físico y psicológico libre de discapacidad, siendo éste el principal problema detectado junto a la preocupación del bienestar de sus familiares y la conciliación de su vida familiar con el rol de cuidadoras que muchas de ellas desempeñan. De la misma manera, cabe decir que más de la mitad de las mujeres estudiadas han aprendido estrategias socioeducativas de afrontamiento activo que les permite resolver las situaciones de crisis de forma efectiva.

En lo que concierne a la vivencia de su participación en centros sociales, si bien hay que ser conscientes de que es manifiestamente mejorable, se debe señalar no obstante que la tradición de asociacionismo se va asentando con el paso del tiempo, creando un entorno y una referencia en este nivel para futuras generaciones. También presentan inquietudes personales y una actitud abierta hacia el aprendizaje, lo que les ha llevado a demandar y continuar recibiendo formación. 
La participación en actividades socioeducativas de los centros les repercute positivamente tanto a nivel individual, mejorando su autoestima, como en una mejor disposición a la realización de actividades en su día a día: a nivel familiar, mejorando la manera que tienen de relacionarse con los miembros de la familia; y a nivel social, buscando el apoyo de los iguales, aumentando el nivel de sus redes sociales y ejercitando su ciudadanía y el sentimiento de integración social. Su participación en los centros les reporta la posibilidad de ampliar su red de relaciones sociales.

Además, las actividades socioeducativas que desarrollan les facilita la posibilidad de reflexionar y de poder evaluarse desde otras miradas que no son las familiares. Este hecho les permite, incluso, la oportunidad de reconstruir sus historias personales y la posibilidad de intercambiar saberes y conocimientos entre ellas mismas, y entre ellas y los educadores. Todo ello significa para muchas mujeres del estudio un proceso gradual de emancipación personal y de libertad hacia la autorrealización que anteriormente no habían tenido ocasión de experimentar.

En lo que respecta a las características del modelo educativo más adecuado para ellas, y que daría respuesta al último de los objetivos propuestos en la investigación, según los resultados obtenidos se puede concluir que las mujeres mayores de los centros sociales que participan en actividades socioeducativas del valle de Ricote de Murcia presentan preferencia por un formato donde se les de protagonismo en la realización de actividades, donde el aprendizaje dialógico sea el referente, donde haya situaciones de diálogo horizontal, interactividad, trabajo colaborativo... Por eso son tan gratificantes, según expresaron en los grupos de discusión, las actividades de grupo que realizan en los centros. Aparece nítidamente la inclinación por entornos de aprendizaje grupales frente a los individuales, ya que,afirman, el grupo refuerza sus posibilidades de interacción y por tanto de aprendizaje y de socialización.

\section{Referencias}

Abellán, A., \& Esparza, C. (2009). Percepción de los españoles sobre distintos aspectos relacionados con los mayores y el envejecimiento. Madrid: IMSERSO.

Agüera, L., Cervilla, J., \& Martín, M. (2006). Psiquiatría geriátrica. Barcelona, España: Masson.

Alcain, M. D., Rubio, M. C., \& Sevillano, A. (2003). Análisis bibliométrico de la producción científica española sobre mujeres mayores. Madrid, España: Portalmayores.

Barefoot, J. C., Maynard, K. E., Beckham, J. C., Brummet, B. H., Hooker, K., \& Siegler, I. C. (1998). Trust, health, and longevity. Journal of Behavior Medicine, 21(6), 517-526. doi: 10.1023/A:1018792528008

Barnés, P. (2005). El asociacionismo de las mujeres en la Región de Murcia. Murcia, España: Consejería de Presidencia.

Boletín Epidemiológico de la Región de Murcia (2010). Esperanza de vida al nacer en la Región de Murcia, 2004-2007. Servicio de Epidemiología, 30, 725.

CARM (2012). Mujeres de la Región de Murcia. Estadística desde la perspectiva de género 2011. Murcia, España: Autor.

Escotorin, P., \& Roche, R. (Eds.) (2011). Cómo y por qué prosocializar la atención sanitaria. Reflexiones, desafíos y propuestas. Barcelona, España: Fundació Universitaria Martí L'humà. 
Fernández-Ballesteros, R. (2009). Envejecimiento activo: Contribuciones de la psicología. Madrid, España: Pirámide.

Hamarat, E., Thompson, D., Steele, D., Matheny, K., \& Simmons, C. (2002). Age differences in coping resources and satisfaction with life among middle-aged, young-old adults. Journal of Genetic Psychology, 163 (3), 360-367. doi: 10.1080/00221320209598689

Hernández Pedreño, M. (2000). Desigualdades según género en la vejez. Murcia, España: Secretaría Sectorial de la Mujer y de la Juventud.

IMSERSO (2011). Envejecimiento activo. Libro Blanco. Madrid: Ministerio de Sanidad, Política Social e Igualdad.

INE (2014). Estimación de la población española actual. Recuperado de www.ine.es

Krzemien, D., Urquijo, S., \& Monchietti, A. (2004). Aprendizaje social y estrategias de afrontamiento a los sucesos críticos del envejecimiento femenino. Psicothema, 16(3), 350-356.

Lehr, U. (2009). Prólogo. En R. Fernández-Ballesteros, Envejecimiento activo (pp. 11-13). Barcelona, España: Pirámide.

Levy, B. (2002). Longevity increased by positive self-perceptions of aging. Journal of Personality and Social Psychology, 83 (2), 261-270. doi: 10.1037/00223514.83.2.261

Levy, B., \& Myers, L. M. (2003). Preventive health behaviors influenced by selfperceptions of aging. Prevention Medical, 39(3), 203-211.

Lecompte, M., \& Goetz, J. (1982). Problems of reliability and validity in etnographic research. Review of Educational Research, 52, 57-76.

Lorenzo, T., Millán-Calenti, J. C., Lorenzo-López, L., \& Maseda, A. (2013). Efectos del programa educativo GERO-HEALTH sobre el nivel de interiorización de conocimientos de prevención y promoción de la salud en personas mayores. Revista de Investigación Educativa, 31(2), 501-516. doi: 10.6018/rie.31.2.163391

Martínez de la Iglesia, J. et al. (2002). Versión española del cuestionario de Yesavage abreviado (GDS) para el despistaje de depresión en mayores de 65 años: Adaptación y validación. Medifam, 12, 620-630.

Martínez de Miguel, S. (2003). Reconstruyendo la educación de personas mayores. Granada: Grupo Editorial Universitario.

Martínez de Miguel, S., \& Escarbajal de Haro, A. (2009). Alternativas socioeducativas para las personas mayores. Madrid, España: Dykinson.

Meléndez, J. C., Navarro, F., Oliver, A., \& Tomás, J. M. (2009). La satisfacción vital en los mayores. Factores sociodemográficos. Boletín de Psicología, 95, $29-42$.

Miñano, L., \& Martínez de Miguel, S. (2011). El asociacionismo y las necesidades socioeducativas en los Centros de Mayores del municipio de Murcia. Revista de Investigación Educativa, 29(2), 325-340.

Montero, I., García Mínguez, J., \& Bedmar, M. (2011). Ciudadanía activa y personas mayores. Contribuciones desde un modelo de educación expresiva. Revista Iberoamericana de Educación, 55(5), 1-13.

Moor, C., Zimprich, D., Schmitt, M., \& Kliegel, M. (2006). Personality, aging selfperceptions, and subjective health: A mediation model. Inernational Journal of Aging Human Development, 63(3), 241-57. doi: 10.2190/AKRY-UMYK-PB1V-PBHF

Pérez Díaz, J. (2006). Demografía y envejecimiento. Recuperado de http://www.imsersomayores.csic.es/documentos/documentos/perez-demografia-01.pdf. 
Pérez Díaz, J. (2010). El envejecimiento de la población Española. Investigación y Ciencia, 410, 34-42.

Pérez Ortiz, L. (2004). Envejecer en femenino. Las mujeres mayores en España a comienzos del siglo XXI. Madrid, España: Instituto de la Mujer.

Puigvert, L. (2005). Las otras mujeres. Barcelona, España: El Roure.

Rodríguez, G. (Ed.) (2013). Las personas mayores que vienen. Autonomía, solidaridad y participación social. Madrid, España: Fundación Pilares.

Sánchez Palacios, C. (2004). Estereotipos negativos hacia la vejez y su relación con variables sociodemográficas, psicosociales y psicológicas (Tesis doctoral). Recuperado de http:// www.biblioteca.uma.es/bbldoc/tesisuma/16704046.pdf

Serdio, C. (2004). Envejecimiento, mujer y educación. Estudio cualitativo sobre la participación educativa de la mujer mayor. Granada, España: Grupo Editorial Universitario.

Serdio, C. (2006). Mujeres que envejecen, mujeres que aprenden. Salamanca, España: Universidad Pontificia de Salamanca.

Solé, C. et al. (2005). La educación en la vejez: Razones para participar en programas educativos y beneficios que se extraen. Revista de Ciencias de la Educación, 203, 453-464.

Triadó, C., \& Villar, F. (1997). Modelos de envejecimiento y percepción de cambios en una muestra de personas mayores. Anales de psicología, 73, 43-55.

Urquijo, S., Monchietti, A., \& Krzemien, D. (2008). Adaptación a la crisis vital del envejecimiento: Rol de los estilos de personalidad y de la apreciación cognitiva en adultas mayores. Anales de psicología, 24(2), 299-311.

Vega, J. L., Buz, J., \& Bueno, B. (2002). Niveles de actividad y participación social en las personas mayores de 60 años. Revista Interuniversitaria de Formación del Profesorado, 45, 33-53.

Villaplana, C. (2010). Relación entre los programas universitarios para mayores y la satisfacción durante la jubilación y la calidad de vida. Revista de Investigación Educativa, 28(1), 195-216. 


\section{ANEXO. GUÍA DE CUESTIONES PARA LOS GRUPOS DE DISCUSIÓN}

\section{a) GUÍA DE CUESTIONES ACERCA DE LA PERCEPCIÓN DE LA VEJEZ Y LA CALIDAD DE VIDA}

1. ¿Qué imagen tienen del envejecimiento en general y cómo ven su propio proceso de envejecimiento?

2. ¿Cómo se ven con respecto a la manera de envejecer de sus madres y cómo creen que envejecerán y se comportarán sus hijas cuando tengan su misma edad?

3. ¿Es posible seguir creciendo y desarrollándose cuando se es mayor?

4. ¿Qué cosas creen que están aportando y qué pueden aportar a la sociedad?

5. ¿Cómo creen que la sociedad ve a las mujeres mayores y qué piensan al respecto?

6. ¿Creen que es necesario trabajar para favorecer o potenciar una imagen más positiva de la mujer mayor?

7. ¿Cómo podríamos hacer para que los problemas que afectan a las mujeres mayores pudieran ser mejor entendidos por la sociedad.

b) GUÍA DE CUESTIONES ACERCA DE LA PARTICIPACIÓN SOCIOEDUCATIVA EN LOS CENTROS SOCIALES

1. Mayoritariamente ninguna de Uds. desea quedarse estancada en la vida. ¿Cómo le podríamos ayudar desde los centros sociales?

2. Para aprender a vivir mejor, ¿qué temas son los que les interesan?

3. ¿Son importantes las actividades culturales y educativas en estas edades?

4. ¿Cómo les gustaría llevar a cabo las actividades socioeducativas?

5. ¿Cómo son los educadores/monitores y cómo les gustaría que fuesen las sesiones?

6. ¿Por qué piensan que las mujeres mayores aprenden cosas diferentes respecto a como lo hacen los hombres?

7. ¿Qué favorece su participación en actividades culturales y educativas?

Fecha de recepción: 26/11/2014.

Fecha de revisión: 26/11/2014.

Fecha de aceptación: 30/04/2015. 